establishment proteins. However, these proteins cannot recognize $G \beta \gamma$ on their own. Instead, the polarity-establishment proteins Cdc24 and Bem1 bind to Far1, which in turn binds to $G \beta \gamma^{4,5}$. In support of a function for Farl in polarity establishment, alleles of both FAR1 and CDC24 have been identified ${ }^{6,7}$, termed farl-s and $c d c 24$ $m$, respectively, that can polarize in response to the internal cell-cycle signal generated during vegetative growth, but fail to polarize properly in response to a gradient of pheromone.

Shimada et al.'s studies ${ }^{3}$ now reveal that Far1 is more than just a linker for assembly of a polarity-establishment complex - it also plays a crucial part in the temporal regulation of polarity. Farl controls polarity temporally by regulating the access of Cdc24 to sites of polarity establishment, so modulating Cdc24's activity. The localization of Cdc24 within the cell changes as a function of cell-cycle position - it is localized to the nucleus in early G1 and to the bud tip from the G1-to-S-phase boundary until mitosis, at which time it becomes localized to the bud neck ${ }^{3,5,8}$. It is Far1 that brings Cdc24 into the nucleus during G1, sequestering it there until a polarity signal is received either from the cell cycle or as a result of activation of the pheromoneresponse pathway.

Regulation of Far1 by a cell-cycle kinase provides the internal cue that triggers movement of Cdc24 to the cytoplasm during vegetative growth, leading to its activation and polarization of the actin cytoskeleton. At the boundary between the G1 and S phases of the cell cycle, Farl is phosphorylated by the G1 cyclin-cyclindependent kinase (CDK) complex (ClnCdc28), inducing degradation of Far1 by a ubiquitin-dependent pathway'. Shimada et $a l^{3}$ show that when Far1 is degraded, Cdc24 is released and exported to the cytoplasm, where it can associate with the nascent bud site through interactions with Bud1. Thus, Far1 anchors Cdc24 in the nucleus during early G1, preventing it from functioning in the cytoplasm until the internal cue derived from the cell cycle is received, signified by activation of Cln-Cdc28.

Regulation of Farl activity in response to pheromones leads to Cdc 24 activation by a mechanism that is completely different from that used to respond to the internal, cellcycle-derived cue ${ }^{3}$. When yeast cells sense a gradient of pheromone, the pheromoneresponse pathway is activated, leading to phosphorylation of Far1 by the MAPK Fus3. The phosphorylated form of Farl is then able to associate with the transport receptor Msn5, which escorts Far 1 out of the nucleus ${ }^{10}$. Intriguingly, $\mathrm{Cdc} 24$ comes along for the ride and thus is exported to the cytoplasm in association with Far1, where it is then targeted to the pheromone-induced site of polarization by the interaction of Far 1 with $G \beta \gamma$. Export of the Cdc24-Far1 complex thus has two consequences: it promotes its association with the polarization site directed by the external pheromone signal, and also prevents Cdc24 from

\title{
Wingless and Naked
}

Patterning of the embryo - the process by which asymmetrically organized cell types and organs are generated from a single cell to make up an individual - is controlled by a surprisingly limited number of signalling pathways (for example, the Hedgehog, Wingless, FGF, TGF- $\beta$ and EGFR pathways). What's amazing is that patterning of structures as different as the limb or appendage and hair or bristles (for vertebrates or invertebrates, respectively), in species as different as the fruitfly Drosophila melanogaster and mammals, is controlled by the same conserved pathways. Specificity is achieved by the fine cross-regulation of these pathways by one another. Negative feedback loops have been described whereby activation of these signalling pathways leads to expression of negative regulators. These inhibitors may directly antagonize the signal, possibly by interfering with ligand binding to the receptor at the start of the signal cascade in the case of soluble inhibitors or membrane inhibitors, or by interfering with the downstream signalling cascade in the case of intracellular inhibitors. Alternatively, inhibitors may generate an antagonizing signal. But, until now, a negative feedback loop had not been described for the Wingless pathway.

Because the phenotype of Drosophila with a loss-of-function mutation in the naked cuticle ( $n k d)$ gene resembles that of wingless (wg) gain-of function mutants and wg transgenic animals, Nkd has been proposed to be an antagonist of $\mathrm{Wg}$ signalling. Genetic evidence also indicated that $n k d$ might be a direct transcriptional repressor of Engrailed (a target of Wg), or might somehow influence Wg transport. Matthew Scott and colleagues (Nature 403, 789-795; 2000) now show that Nkd indeed opposes the effect of a Wg signal. The nkd mutation, which is lethal in the embryo and causes multiple segmental defects, has been described previously and named after the absence of denticles in the mutant Drosophila embryos. Scott and colleagues have now cloned the nkd gene, and show that its expression pattern parallels that of $w g$ in the Drosophila embryo and larva, indicating that $n k d$ may be a target for $\mathrm{Wg}$. They confirm this by monitoring the expression of $n k d$ in gain-of-function and loss-of-function wg mutants. Overexpression of $n k d$ (right-hand panel in figure below) and decreased wg activity (middle panel) in the Drosophila larva produce similar adult phenotypes (such as the absence of wings in the middle and right-hand panels). Scott et al.'s results indicate that Nkd interferes with Wg signalling, which they also show to be the case for Wnt signalling in the frog Xenopus laevis. So, as is the case for Hedgehog signalling with Patched, EGFR signalling with Argos, Kekkon and Sprouty, and TGF- $\beta$ signalling with Dad, Wg signalling now has its own inhibitor and its own negative feedback loop.

The question now will be how Nkd inhibits Wg signalling. The evidence so far is that it does not, at least initially, interfere with the expression or transport of Wg, and so it most likely interferes with the signalling cascade downstream of the Wg receptors, Frizzled and Dishevelled. But where in the cascade, and how, does it act? Nkd bears a region of homology to calciumbinding EF-hand domains, which might provide a hint to its mode of action. However, Nkd seems to lack other structural features of EF-hand-containing proteins such as EF-hand repeats or myristoylation sequences, so this clue might be misleading.

VALERIE DEPRAETERE
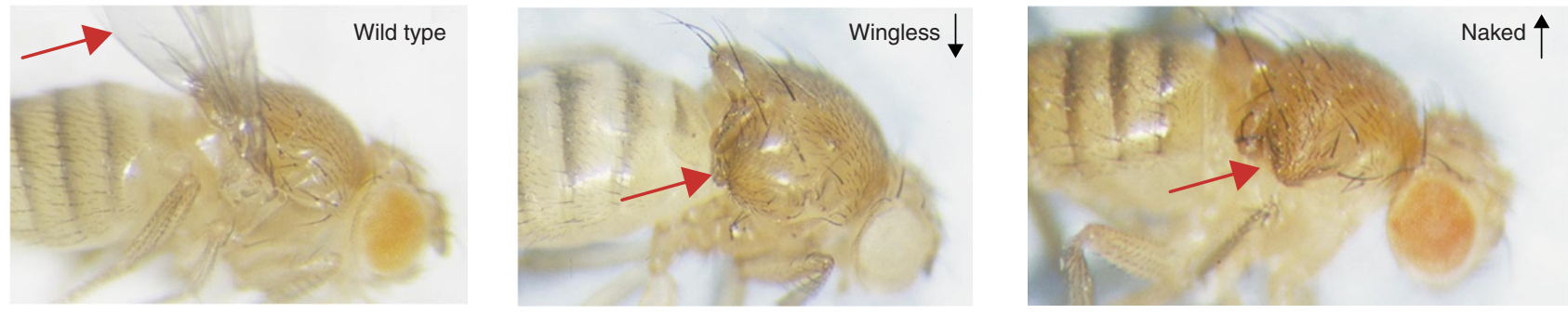